\title{
Optimizing GHT-Based Heart Localization in an Automatic Segmentation Chain
}

\author{
Axel Saalbach ${ }^{1}$, Irina Wächter-Stehle ${ }^{1}$, Reinhard Kneser ${ }^{2}$, \\ Sabine Mollus ${ }^{2}$, Jochen Peters ${ }^{1}$, and Jürgen Weese ${ }^{1}$ \\ ${ }^{1}$ Philips Research, Röntgenstrasse 24 - 26, 22335 Hamburg, Germany \\ 2 Philips Research, Weisshausstrasse 2, 52066 Aachen, Germany \\ axel.saalbach@philips.com
}

\begin{abstract}
With automated image analysis tools entering rapidly the clinical practice, the demands regarding reliability, accuracy, and speed are strongly increasing. Systematic testing approaches to determine optimal parameter settings and to select algorithm design variants become essential in this context. We present an approach to optimize organ localization in a complex segmentation chain consisting of organ localization, parametric organ model adaptation, and deformable adaptation. In particular, we consider the Generalized Hough Transformation (GHT) and 3D heart segmentation in Computed Tomography Angiography (CTA) images. We rate the performance of our GHT variant by the initialization error and by computation time. Systematic parameter testing on a compute cluster allows to identify a parametrization with a good tradeoff between reliability and speed. This is achieved with coarse image sampling, a coarse Hough space resolution and a filtering step that we introduced to remove unspecific edges. Finally we show that optimization of the GHT parametrization results in a segmentation chain with reduced failure rates.
\end{abstract}

Keywords: GHT, heart localization, heart segmentation, CTA.

\section{Introduction}

It is well known that the performance of image processing and analysis algorithms in terms of reliability, accuracy, and speed can strongly depend on parameter settings and design variants. Systematic approaches to determine optimal parameter settings and algorithm design variants are one approach to address this challenge. In general, the algorithm performance can be optimized by systematically testing parameter value combinations on image databases and selecting those values that minimize a metric of the processing result as used during validation. Hautvast et al. 1], for instance, performed full factorial experiments and analyzed the results using the technique of analysis of variances (ANOVA) to optimize parameter settings of their cardiac MR contour segmentation.

Complex image processing algorithms such as fully automatic heart segmentation [23] depend on many design decisions and parameter settings, making

G. Fichtinger, A. Martel, and T. Peters (Eds.): MICCAI 2011, Part III, LNCS 6893, pp. 463470, 2011. (C) Springer-Verlag Berlin Heidelberg 2011 
straightforward optimization difficult. On the one hand, due to processing time demands, an extensive optimization of an algorithm is often not feasible, even when large computing recourses are available. Tools from experimental design 4. can help to efficiently identify relevant parameter combinations and find optimal parameter values with limited effort. On the other hand, the influence of a single parameter or design variant may have a negligible influence on a metric characterizing the overall processing result. Therefore, approaches for the optimization of individual parts of a complex processing chain are important. An example is Simulated Search [5] allowing to select optimal boundary detection functions per triangle in model-based segmentation.

We present an approach to optimize the initial organ localization step in a complex segmentation chain consisting of organ localization, parametric organ model adaptation, and deformable adaptation. In particular we consider the Generalized Hough Transformation (GHT) 6] for 3D localization and model-based heart segmentation in CTA images. We show that reliability and computational speed of our GHT variant can be optimized by systematic parameter testing using a metric for the initialization error resulting in an improved overall segmentation chain.

In the following section, the segmentation chain is outlined, our GHT variant is described and parameter optimization is introduced. Section 3 describes the experiments and section 4 presents our conclusions.

\section{Methods}

\subsection{Segmentation Chain}

Automatic heart segmentation can be done in four steps. First, the heart is localized with a GHT and the heart model is placed in the image. Second, pose and scaling of the heart model are adjusted by parametric adaptation. Third, the relative sizes of the heart chambers are adjusted using a parametric adaptation with a piece-wise affine transformation. Finally, accurate adaptation is performed using shape constrained deformable models.

Fig. 1 shows how adaptation of the heart model to the image improves in the individual steps. All steps are important, because each step relies on a sufficiently accurate initialization provided by the previous step [2 5]. Initial heart localization is very crucial as the entire segmentation chain fails, if heart localization fails or is too inaccurate.

\subsection{GHT Algorithm}

The GHT [6] is a robust and powerful method for detecting a pre-defined shape undergoing geometric transformations in an image. During learning, a description of the target shape is encoded into a reference table also known as R-table. The entries of the R-table are vectors pointing from shape boundary points to a reference point. These vectors are binned by the orientation of the boundary point. For detecting the position of the shape in a new image, edges are determined. For each detected edge element, the gradient orientation is measured 


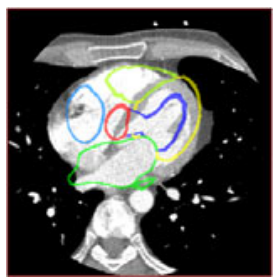

(a)

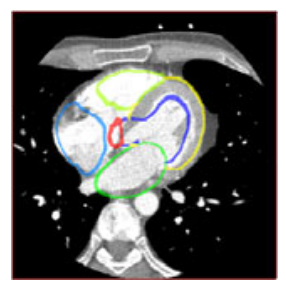

(b)

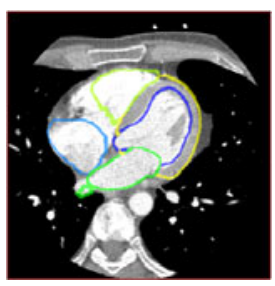

(c)

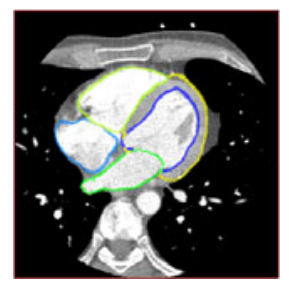

(d)

Fig. 1. Heart mesh after GHT-based localization (a), parametric adaptation with a similarity (b) and piecewise-affine (c) transformation and after deformable adaptation (d) overlaid onto a CTA image.

yielding an index for an R-table entry, and the positions pointed by all vectors under this entry are incremented in an accumulator array. This process is called voting and the accumulator array is also referred to as Hough space. The final shape location is given by the highest peak in the accumulator array. Next to translations, further degrees of freedom such as rotation and scaling can be handled by increasing the dimension of the Hough space.

Our GHT implementation can be considered as a 3D variant of the Shape Variant Hough Transform proposed by Brejl and Sonka [7, while we focus in this application on the determination of translations and scaling levels. In order to take variations in heart shape and orientation into account, images and corresponding reference meshes of $N_{\text {train }}$ individuals were used for training. The center of gravity of the individual meshes is used to establish a common reference point. Fig. 2 shows the heart model and a flow chart of our GHT algorithm which offers multiple options and parameter settings as described in the following.

For our GHT algorithm, training starts by estimating the image gradient orientation at the triangle centers of the $N_{\text {train }}$ heart meshes, which can be done with a $3 \times 3 \times 3$ Sobel operator or a Canny filter. While Brejl and Sonka [7] used a border appearance model to control the voting process, we use multiple independent filters to identify specific edges. Triangles with weak gradients may be filtered out by defining a threshold $t_{\text {weak edge }}$ for the gradient magnitude that removes the fraction $f_{\text {weak edge }}$ of triangles with the smallest gradient magnitude from R-table construction. In addition, the average gray-value is determined in a $3 \times 3 \times 3$ neighborhood around a triangle center and a desired gray-value range $\left[g_{\text {edge,min }}, g_{\text {edge,max }}\right]$ for an edge can be defined. Edges with an average grayvalue outside this range are also excluded from R-table construction. Gradient computation as well as gray-value analysis can be done in combination with image sub-sampling.

The normalized image gradient is used to assign the triangle centers of the $N_{\text {train }}$ heart meshes to bins of the R-table. For the definition of the bins we use a simple tiling of a spherical surface with rectilinear cells. Here, the size of the cells is specified by means of the angular resolution $\phi$. In order to obtain cells with a similar size, we adapt the value of $\phi$ over the sphere. Furthermore, a merging 


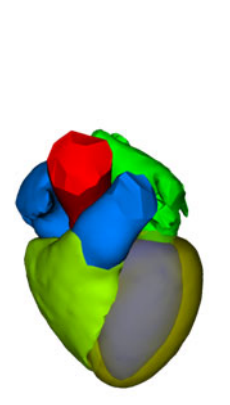

(a)

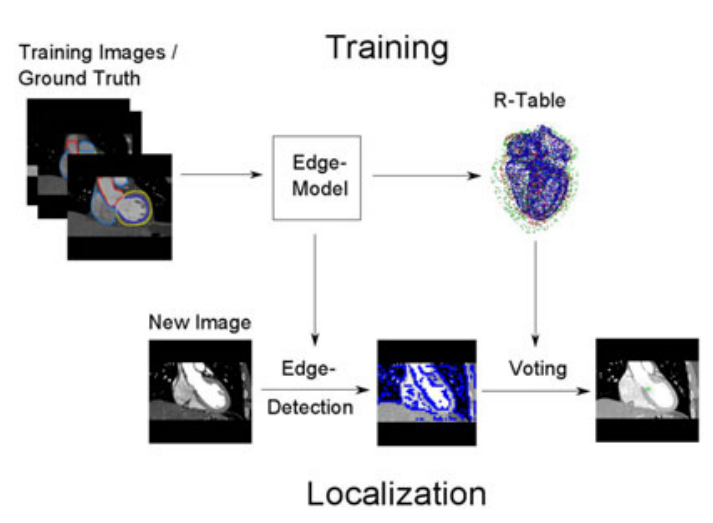

(b)

Fig. 2. Heart model (a) and flow chart of our GHT algorithm (b)

step can be applied to reduce the number of R-table entries. For that purpose, an Euclidean grid with edge length $d_{\text {merge }}$ is generated and triangle centers of the $N_{\text {train }}$ heart meshes in a cell of the grid that belong to the same R-table bin are replaced by a single R-table entry and a weight $w_{\mathrm{R}, i}$ defining the frequency of the entry. In a subsequent pruning step, R-table entries can be removed if their weight $w_{\mathrm{R}, i}$ is smaller than a threshold $w_{\mathrm{R}, \text { Threshold }}$.

For localization, the same pre-processing is applied that has been used for training, i.e. the gradient computation can either be done with a Sobel operator or the Canny filter. Weak edges with a gradient magnitude smaller than the threshold $t_{\text {weak edge }}$ as well as edges with an average gray-value outside the interval $\left[g_{\text {edge,min }}, g_{\text {edge,max }}\right]$ can be filtered out. Gradient computation and grayvalue analysis can be done in combination with sub-sampling. With regard to the voting and the model point weights, in recent years several extensions of the GHT have been proposed (see e.g. [8] and [9]). For simplicity, we consider two basic variants only, voting may use the weights $w_{\mathrm{R}, i}$ or all R-table entries may be attributed the same weight.

\subsection{GHT Optimization}

Optimization of the GHT parameters and the analysis of design variants require the definition of a suitable metric. In our application the result of the GHT is used to position a (transformed) heart model. In order to quantify how well the this model is positioned in the image, we use the mean constrained point-tosurface distance [2]:

$$
\epsilon_{\mathrm{GHT}}=\frac{1}{M} \sum_{i=1}^{M} \frac{\epsilon_{\Pi(r)}\left(c_{i}^{G H T}, c_{i}^{r e f}\right)+\epsilon_{\Pi(r)}\left(c_{i}^{r e f}, c_{i}^{G H T}\right)}{2},
$$

with $M$ as the number of triangles and $\epsilon_{\Pi(r)}\left(c_{i}^{G H T}, c_{i}^{r e f}\right)$ as the smallest Euclidean distance between the triangle center of the GHT initialized mesh and 
a corresponding surface patch in the reference mesh. Here, the surface patch is restricted to triangles within a small geodesic radius $(r=10.00 \mathrm{~mm})$ around $c_{i}^{r e f}$. If the initialization error $\epsilon_{\mathrm{GHT}}$ is small, the GHT-based model placement is already close to the desired result. Otherwise, the following adaptation steps may fail, and a segmentation failure may be obtained.

To carry out GHT parameter optimization and to analyze design variants, different GHTs were trained and evaluated. In order to access the generalization capabilities of the different variants, and to avoid a biased analysis, 10-fold cross validation was employed. Next to the initialization error $\epsilon_{\mathrm{GHT}}$, we recorded the computation time for GHT-based heart localization. Systematic testing of parameter value combinations was done by grid search.

\section{Experiments}

\subsection{Tested Parameter Values}

For testing different GHT parameterizations, we used a database with $N_{\text {train }}=$ 89 cardiac CTA images with corresponding ground truth annotations in terms of reference meshes. For the generation of the 3D ground truth annotations, automatic segmentations were inspected and refined by a domain expert.

Edge detection was done with the Sobel or the Canny filter. To remove nonspecific edges, the threshold $t_{\text {weak edge }}$ was determined to discard the $f_{\text {weak edge }}=$ $5 \%$ weakest edges. In addition, removal of dark edges was tested, i.e. $g_{\text {edge min }}$ was determined in a way that $90 \%$ or $95 \%$ of the triangles have a larger average gray-value. For image sampling, sub-sampling with 2.0, 3.0, 4.0, and $5.0 \mathrm{~mm}$ was considered. An angular resolution of $\phi=4.0,6.0$, and $8.0 \mathrm{deg}$ was used for the R-table. Merging was offered using an Euclidean edge length matched with image sub-sampling. For pruning, the thresholds $w_{\mathrm{R} \text {,Threshold }}=0.05 \times N_{\text {Train }}$ and $0.10 \times N_{\text {Train }}$ were tested. During localization, three scaling levels $(0.8,1.0$, and 1.2) were used. The resolution of the Hough space was matched to the sub-sampling rate for edge detection and gray-value analysis. Voting was tested using the weights $w_{\mathrm{R}, i}$ resulting from merging, and identical weights for all Rtable entries. In total, this results in 1680 different parametrizations, resp. design variants for the GHT. For a comprehensive evaluation of all combinations, cross validation experiments were carried out using a Windows based compute cluster $(5 \times$ Dell R710 with $2 \times \operatorname{Intel}(\mathrm{R})$ Xeon(R) CPU Six Core, X5680 @3.3 GHz, 12 MB cache, 12 GB RAM; Windows HPC Server 2008).

\subsection{Parameter Dependence of the GHT}

The evaluation of the different parameterizations revealed a broad range of variations with regard to computation time as well as localization accuracy. Fig. 3 shows a subset of the results as a scatter plot, where each dot refers to the average outcome in the 89 CTA images evaluated via 10-fold cross validation for one parametrization. While a large number of different parameterizations resulted in a reasonable localization accuracy, especially the variation in computation time 


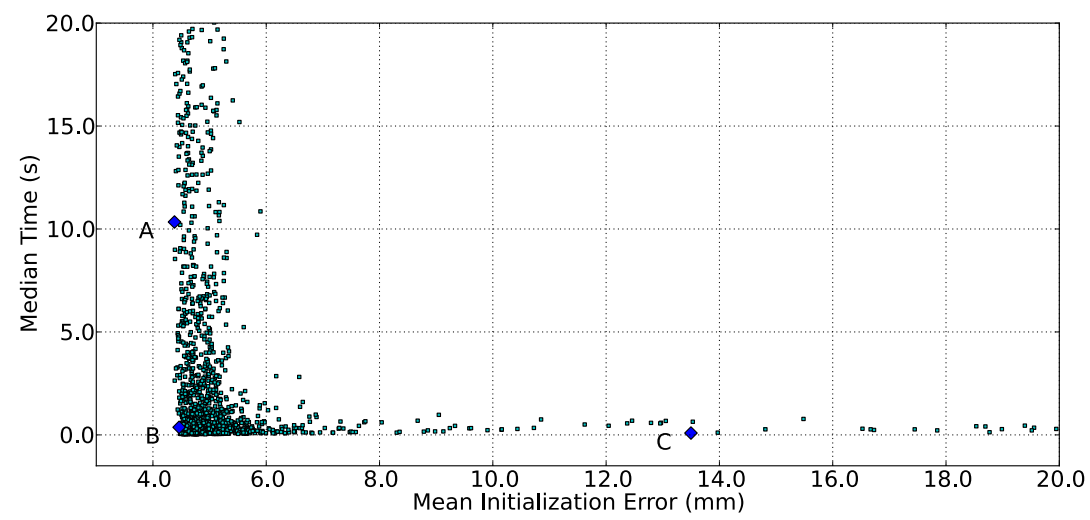

Fig. 3. Overview of the GHT-based localization results. The diagrams show the outcome for different parameterizations in terms of (median) computation time and (average) initialization error $\epsilon_{\mathrm{GHT}}$. Three specific configurations have been highlighted (see also section 3.3$)$.

is huge. The scatter plot also shows that there are parameterizations with a good localization accuracy that are very fast at the same time.

Looking in more details at the parameterizations, the results show that a good localization of the heart can be achieved already with coarse image sampling and a coarse resolution of the accumulator (both $5 \mathrm{~mm}$ ). This is of advantage from a computational perspective as well as for the memory consumption. Merging and pruning did allow to reduce the R-table size and increases the localization speed, while the localization accuracy is only slightly decreased. Voting with identical weights typically outperformed voting with the weights $w_{\mathrm{R}, i}$ from merging.

Even though the GHT turned out to be quite robust with respect to the employed edge detection techniques, the best results (in terms of accuracy and speed) were achieved by means of a combination of a Canny filter and grayvalue based acceptance intervals. Both techniques remove unspecific edges which resulted in an increased speed and localization accuracy. However, the analysis showed also that an appropriate combination of these techniques is essential. A very restrictive edge detection strategy resulted often in a poor localization accuracy since too many edges were removed from the voting process. This shows that systematic testing is crucial to identify good parameter value combinations.

\subsection{Influence of the GHT on Heart Segmentation}

Finally, the influence of different GHT parameterizations on the heart segmentation was investigated. This experiment was carried out with 242 CTA datasets that were independent from the data sets used in section 3.1 and 3.2. These datasets represent a broad spectrum of diseases and image quality, and have been acquired with scanners from different vendors at multiple sites. For the evaluation, reference segmentations were obtained by means of a parametric 
model adaptation followed by a deformable adaptation. In order to achieve an accurate localization, that is independent from the GHT approach, a manual initialization of the heart was performed by a domain expert using three landmarks (apex, center of mitral valve, center of aortic valve).

Three different parameterizations of the GHT were used in these experiments. They are indicated by A (accurate / slow), B (accurate / fast) and C (inaccurate / fast) in the scatter plot of Fig. 3. These GHT parameterizations were used to initialize the segmentation chain and the resulting segmentation was compared with the reference segmentation. A segmentation was classified as success if the constrained point-to-surface error was $<2 \mathrm{~mm}$ and as a failure otherwise. Table 1 summarizes the results. Fig. 4 shows examples of successful segmentations.

Table 1. Influence of the GHT parametrization on the segmentation chain. The initialization error and the median time for localization refer to Fig. 3. Segmentations with a constrained point-to-surface error $>2 \mathrm{~mm}$ were classified as failure.

\begin{tabular}{l|r|r|rr} 
GHT parameterization & Initial. Error & Median Time & \multicolumn{2}{|c}{ CT failures } \\
\hline \hline (A) accurate / slow & $4.38 \mathrm{~mm}$ & $10.3 \mathrm{sec}$ & $4 / 242(1.7 \%)$ \\
(B) accurate / fast & $4.46 \mathrm{~mm}$ & $0.4 \mathrm{sec}$ & $6 / 242 \quad(2.5 \%)$ \\
(C) inaccurate / fast & $13.50 \mathrm{~mm}$ & $0.1 \mathrm{sec}$ & $90 / 242(38.0 \%)$ \\
\hline
\end{tabular}

According to Table 1, an improper GHT parametrization can lead to a very poor performance of the entire segmentation chain. With a suitable GHT parametrization, the heart can be detected in a fraction of a second and a failure rate of $2-3 \%$ of the entire segmentation chain can be achieved. When accepting a computation time in the order of $10 \mathrm{~s}$ for heart localization, the failure rate can be slightly reduced.

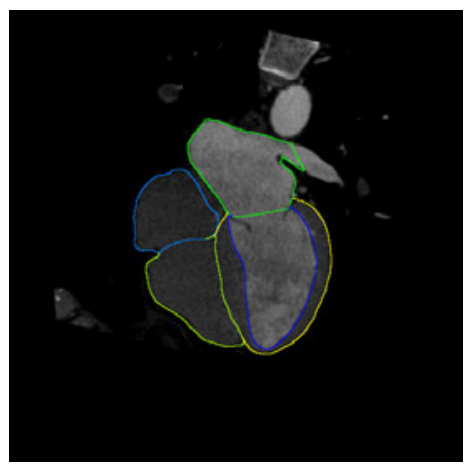

(a)

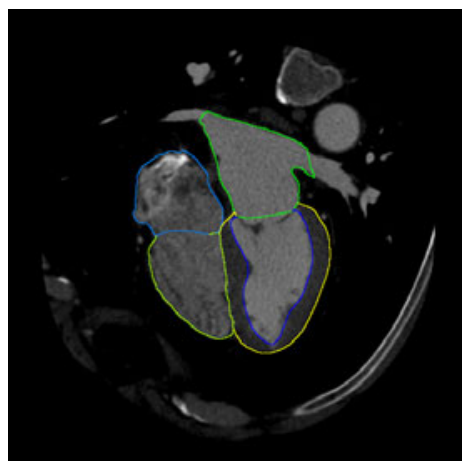

(b)

Fig. 4. Example segmentations for two CTA images 


\section{Conclusions}

We presented an approach to optimize the initial organ localization step in a complex segmentation chain consisting of organ localization, parametric organ model adaptation, and deformable model adaptation. In particular, we considered the Generalized Hough Transformation (GHT) for heart localization and heart segmentation in CTA. The GHT performance was measured in terms of the initialization error and computation time. By systematic parameter testing on a computing cluster we identified a GHT parametrization allowing fast and reliable heart localization. A good performance was achieved with coarse image sampling $(5 \mathrm{~mm})$, a coarse Hough space resolution $(5 \mathrm{~mm})$ and filtering to remove unspecific edges. Finally, we showed that a failure rate for automatic heart segmentation in CTA images of only $2-3 \%$ can be achieved with a suitable GHT parametrization.

\section{References}

1. Hautvast, G., Lobregt, S., Breeuwer, M., Gerritsen, F.: Automatic contour propagation in cine cardiac magnetic resonance images. IEEE Transactions on Medical Imaging 25(11), 1472-1482 (2006)

2. Ecabert, O., Peters, J., Schramm, H., Lorenz, C., von Berg, J., Walker, M., Vembar, M., Olszewski, M., Subramanyan, K., Lavi, G., Weese, J.: Automatic ModelBased segmentation of the heart in CT images. IEEE Transactions on Medical Imaging 27(9), 1189-1201 (2008)

3. Zheng, Y., Barbu, A., Georgescu, B., Scheuering, M., Comaniciu, D.: Four-Chamber heart modeling and automatic segmentation for 3-D cardiac CT volumes using marginal space learning and steerable features. IEEE Transactions on Medical Imaging 27(11), 1668-1681 (2008)

4. Wu, C.F.J., Hamada, M.S.: Experiments: Planning, Analysis, and Optimization, 2nd edn. Wiley, Chichester (2009)

5. Peters, J., Ecabert, O., Meyer, C., Kneser, R., Weese, J.: Optimizing boundary detection via simulated search with applications to multi-modal heart segmentation. Medical Image Analysis 14(1), 70-84 (2010)

6. Ballard, D.: Generalizing the Hough transform to detect arbitrary shapes. Pattern Recognition 13(2), 111-122 (1981)

7. Brejl, M., Sonka, M.: Object localization and border detection criteria design in edge-based image segmentation: Automated learning from examples. IEEE Transactions on Medical Imaging 19(10), 973-985 (2000)

8. Maji, S., Malik, J.: Object detection using a max-margin hough transform. In: IEEE Computer Society Conference on Computer Vision and Pattern Recognition, pp. 1038-1045 (2009)

9. Leibe, B., Leonardis, A., Schiele, B.: Combined object categorization and segmentation with an implicit shape model. In: Workshop on Statistical Learning in Computer Vision, European Conference on Computer Vision, pp. 17-32 (2004) 